\title{
Results Concerning the Trace of Some Biadditive Mappings of Prime and Semiprime Rings
}

\author{
Eqbal Jabur Harjan \\ Department of Mathematic, College of Education, Al-Mustansiriyah University, Baghdad-Iraq.
}

\begin{abstract}
The purpose of this paper is to present some results concerning the trace of symmetric $(\alpha, \alpha)$ Biderivation and symmetric left $\alpha$-Bimultiplier on prime rings. In these results we investigate commutativity of rings, further some certain identities satisfying by symmetric $(\alpha, \alpha)$-Biderivation and biadditive mappings that make these mapping $\alpha$-commuting.
\end{abstract}

Keywords: Prime rings, Trace of biadditive mappings, Symmetric $(\alpha, \alpha)$-Biderivation, Symmetric left $\alpha$-Bimultiplier, $\alpha$-commuting mappings.

\section{Introduction}

Throughout this discussion, unless otherwise mentioned $R$ will represent an associative prime ring with center $Z(R)$ and $\alpha$, $\tau \in \operatorname{Aut}(R)$. For $x, y \in R$, the symbol $[x, y]$ will denote the commutator $x y-y x$. A ring $R$ is called 2-torsion free, if $2 x=0, x \in R$, implies $x=0$. Recall that $R$ is prime if for any $a, b \in R$, $a R b=\{0\}$ implies $a=0$ or $b=0$ and semiprime if for any $a \in R, a R a=\{0\}$ implies $a=0$.

In [1], T. K. Lee introduce the notion of $\alpha$ commuting mappings in the following way: A mapping $\varphi: R \rightarrow R$ is said to be $\alpha$-centralizing on $R$ if $[\varphi(x), \alpha(x)] \in Z(R)$, for all $x \in R$. In special case when $[\varphi(x), \alpha(x)]=0$, for all $x \in R$, the mapping $\varphi$ is called $\alpha$-commuting. If $\varphi(x)$ $a(x)+a(x) \varphi(x)=0$ holds for all $x \in U$, then $\varphi$ is said to be skew $\alpha$-commuting

A mapping $\mathcal{B}: R \times R \rightarrow R$ is called symmetric if $\mathcal{B}(x, y)=\mathcal{B}(y, x)$ for all pairs $x, y \in R$. A mapping $f: R \rightarrow R$ defined by $f(x)=\mathcal{B}(x, x)$, where $\mathcal{B}$ is a symmetric mapping will be called the trace of $\mathcal{B}$. It obvious that in case $\mathcal{B}$ is a symmetric mapping which is also biadditive (i.e., additive in both arguments), the trace of $\mathcal{B}$ satisfies $f(x+y)=f(x)+2 \mathcal{B}(x, y)+f(y)$, for all $x, y \in R$. The notion of symmetric Biderivation was introduced by Maksa in [2]. A symmetric biadditive mapping $D(.,):. R \times R \rightarrow R$ is called symmetric Biderivation if $D(x y, z)=D(x, z) y+$ $x D(y, z)$ holds all $x, y, z \in R$. If $D$ satisfies that $D\left(x^{2}, z\right)=D(x, z) x+x D(x, z)$ for all $x, y \in R$, then $D$ is said to be symmetric Jordan Biderivation. In 2007 Y. Ceven, and M. A. Öztürk in [3] introduce the concept of symmetric $(\alpha, \tau)$-Biderivation as follows: $\mathrm{A}$ symmetric biadditive mappings $F(.,$.$) :$
$R \times R \rightarrow R$ is called said to be a symmetric $(\alpha, \tau)$-Biderivation if $F(x y, z)=F(x, z) \alpha(y)+$ $\tau(x) F(y, z)$, for all $x, y, z \in R$. Obviously, in this case the relation $F(x, y z)=F(x, y) d(z)+\tau(y)$ $F(x, y)$ is also satisfied for all $x, y, z \in R$. M. Ashraf in 2010 [4], introduced the notion of symmetric generalized $(\alpha, \tau)$-Biderivation as follows: A symmetric biadditive mapping $G(.,):. R \times R \rightarrow R$ is symmetric generalized $(\alpha, \tau)$-Biderivation if there exist symmetric $(\alpha, \tau)$-Biderivation $D$ such that $G(x z, y)=G(x, y) \alpha(z)+\tau(x) D(z, y)$, for all $x, y$, $z \in R$. In case $\alpha=\tau$ the mappings $F$ and $G$ are said to be a symmetric $(\alpha, \alpha)$-Biderivation and symmetric generalized $(\alpha, \alpha)$-Biderivation respectively. A Symmetric biadditive mapping $\mathcal{T}: R \times R \longrightarrow R$ is called a Symmetric left (right) $\alpha$-Bimultiplier where is a homomorphism of $R$ if:

$\mathcal{T}(x z, y)=\mathcal{T}(x, y)(z)(\mathcal{T}(x z, y)=\alpha(x) \mathcal{T}(z, y))$, holds for all $x, y, z \in R$.

The mapping $\mathcal{T}$ is called a Symmetric $\alpha$-Bimultiplier if it is both Symmetric left and right $\alpha$-Bimultiplier (see [5]).

Over the last five decades, many authors $[6,7,8]$ present several results concerning the relationship between the commutativity of prime and semiprime rings and the existence of specific types of a nonzero symmetric generalized $(\alpha, \tau)$-Biderivation and affiliated mappings. In this paper many results of this kind was presented. We shall also briefly discuses of the notion of $\alpha$-commuting mappings. 


\section{Some Preliminaries}

We shall do a great of calculations with commutators, routinely using the following basic identities (see [2]):

$[x y, z]=[x, z] y+x[y, z] \&$

$[x, y z]=[x, y] z+y[x, z]$, for all $x, y, z \in R$.

We state the following well-known results which will be useful in the sequel.

\section{Lemma (2.1): [9]}

Let $R$ be a prime ring of characteristic different from 2 and $\mathcal{J}$ be a nonzero ideal of $R$. let $a, b$ be fixed elements of $R$. if $a x b+b x a=0$ is fulfilled for all $x \in \mathcal{J}$, then either $a=0$ or $b=0$.

\section{Lemma (2.2): [10]}

Let $R$ be semiprime ring, $\mathcal{J}$ a right ideal of $R$. If $\mathcal{J}$ is a commutative as a ring, then $\mathcal{J} \subset Z(R)$. In addition if $R$ is a prime, then $R$ must be commutative.

\section{$\underline{\operatorname{Lemma}(2.3)}:$ [11]}

Let $R$ be a prime ring, and $\mathcal{J}$ be a nonzero left ideal of $R$. If a $(\sigma, \tau)$-Biderivation $D: R \times R \rightarrow R$ satisfies that $D(\mathcal{J}, \mathcal{J})=0$, then $D=0$.

Also, we need to prove the following lemma.

\section{Lemma (2.4):}

Let $U$ be a nonzero left ideal in a 2 -torsion free prime ring $R$. If a symmetric $(\alpha, \alpha)$ Biderivation $F: R \times R \rightarrow R$ has a zero Trace on $U$, then $R$ is commutative or $F$ is zero on $R$.

\section{Proof:}

Let $f$ be the Trace of $F$, then $f(u)=0$, for all $u \in U$.

The linearization of above relation leads because of the 2-torsionity free of $R$ to:

$F(u, \omega)=0$, for all $u, \omega \in U$.

Consequently, for any $r, s \in R$, we have:

$F(r u, s \omega)=0$, for all $u, \omega \in U$.

We shall compute (1) in two different ways to get:

$F(s, r)(\omega) \alpha(u)=0$, for $u, \omega \in U$ and $r, s \in R$. . (2) $F(s, r)(u) \alpha(\omega)=0$, for $u, \omega \in U$ and $r, s \in R$. . (3)
Subtracting (2) from (3) implies that:

$F(s, r)[\alpha(u), \alpha(\omega)]=0$, for $u, \omega \in U, r, s \in R$.

Putting st instead of $s$ in (4), using (4), we arrive at:

$F(s, r)(t) \alpha([u, \omega])=0$, for $u, \omega \in U, r, s, t \in R$.

By primeness of $R$ yields that either $F(s, r)=0$, for all $r, s \in R$, that is $F$ is zero on $R$ or $\alpha([u, \omega])=0$ and consequently $[u, \omega]=0$, for all $u, \omega \in U$.

If $[u, \omega]=0$, for all $u, \omega \in U$ then an application of Lemma (2.2) yields that $R$ is commutative.

\section{The Main Results}

We start our main results with following theorem which looking for the conditions that forces the prime ring $R$ to be commutative.

\section{Theorem (3.1):}

Let $R$ be a 2 -torsion free prime ring and $D: R \times R \rightarrow R$ be a nonzero Symmetric Jordan Biderivation such that $x y-y d(x)=y x-x d(y)$, for all $x, y \in R$, where $d$ is the Trace of $D$, then $R$ is commutative.

\section{Proof:}

Form our hypothesis, we see:

$[x, y]=y d(x)-x d(y)$, for all $x, y \in R$.

The linearization of above relation with respect $x$, we get:

$[x, y]+[z, y]=y d(x)+y d(z)+2 y D(x, z)-x d(y)$ - $z d(y)$, for all $x, y, z \in R$.

In view of (1), and 2-torsionity free of $R$, the above relation reduces to:

$y D(x, z)=0$, for all $x, y, z \in R$.

Now, the substitution $x^{2}$ for $x$ leads to:

$y D(x, z) x+y x D(x, z)=0$, for all $x, y, z \in R$.

According to (2), we have:

$y x D(x, z)=0$, for all $x, y, z \in R$.

Also, the left multiplication of (2) by $x$, we get:

$x y D(x, z)=0$, for all $x, y, z \in R$.

Combining (3) and (4), implies to: 
$[x, y] D(x, z)=0$, for all $x, y, z \in R$.

Replacing $y$ by $y r$ in (5), using (5), leads to: $[x, y] r D(x, z)=0$, for all $x, y, r, z \in R$.

Now, define $\mathcal{K}=\{x \in R: D(x, z)=0$, for all $z \in R\}$ $\mathcal{H}=\{x \in R:[x, y]=0$, for all $y \in R\}$

Then $\mathcal{K}$ and $\mathcal{H}$ are two disjoint sub group of $R$ satisfies that there union equal to $R$, which contradicts Brauer's trick. Since $D$ is a nonzero Jordan Biderivation, we conclude that:

$[\mathrm{x}, \mathrm{y}]=0$, for all $\mathrm{x}, \mathrm{y} \in \mathrm{R}$.

Hence $R$ is a commutative ring.

\section{Theorem (3.2):}

Let $R$ be a 2-torsion free ring and $\alpha$ be an automorphism on $R$. if a symmetric $(\alpha, \alpha)$-Biderivation $F: \quad R \times R \rightarrow R \quad$ satisfies $(x y)-f(x y)=a(y x)-f(y x)$, for all $x, y \in R$, where $f$ is the Trace of $F$, then $R$ is commutative.

\section{Proof:}

For any $x, y \in R$, we have:

$$
\begin{aligned}
& {[a(x), a(y)]=f(x y)-f(y x)} \\
& \quad=\left[a(x)^{2}, f(y)\right]+\left[f(x), \alpha(y)^{2}\right]+2 \alpha(x)
\end{aligned}
$$

$F(x, y) \alpha(y)-2 \alpha(y) F(x, y) \alpha(x)$.

The substitution $x+y$ for $x$ in (1), we get:

$[\alpha(x), \alpha(y)]=\left[\alpha(x)^{2}, f(y)\right]+[\alpha(x) \alpha(y), f(y)]+$ $[\alpha(y) \alpha(x), f(y)]+\left[f(x), \alpha(y)^{2}\right]+2\left[F(x, y), \alpha(y)^{2}\right]$ $+2 \alpha(x) F(x, y) \alpha(y)+2 \alpha(x) f(y) \alpha(y)-2 \alpha(y) F(x$, y) $\alpha(x)-2 \alpha(y) f(y) \alpha(x)$, for all $x, y \in R$. to:

In view of (1), the above relation reduces $[\alpha(x)(y), f(y)]+[\alpha(y) \alpha(x), f(y)]+2[F(x, y)$, $\left.\alpha(y)^{2}\right]+2 \alpha(x) f(y) \alpha(y)-2 \alpha(y) f(y) \alpha(x)=0$.

Again, taking $x+y$ instead of $x$ in (2) and using (2) imply that:

$2\left(\left[\alpha(x)^{2}, f(y)\right]+\left[f(x), \alpha(y)^{2}\right]+2 \alpha(x) F(x, y) \alpha(y)-\right.$ $2 \alpha(y) F(x, y) \alpha(x))=0$, for all $x, y \in R$.

Using the 2-torsionity free of $R$ and relation (1), we arrive at:

$[\alpha(x), \alpha(y)]=\alpha([x, y])=0$, for all $x, y \in R$.
Using the fact that $\alpha$ is an automorphism on $R$, we see:

$[x, y]=0$, for all $x, y \in R$.

Hence $R$ is commutative.

In similar manner we can prove the following theorem.

\section{Theorem (3.3):}

Let $R$ be a 2-torsion free ring and $\alpha$ be an automorphism on $R$. if a symmetric $(\alpha, \alpha)$ Biderivation $F: R \times R \rightarrow R$ satisfies $(x y)+f(x y)=$ $\alpha(y x)+f(y x)$, for all $x, y \in R$, where $f$ is the Trace of $F$, then $R$ is a commutative ring.

\section{Theorem (3.4):}

Let $R$ be a non-commutative 2 -torsion free prime ring and $F: R \times R \rightarrow R$ be a symmetric $(\alpha, \alpha)$-Biderivation. If the Trace $f$ of $F$ is skew $\alpha$-commuting on a nonzero ideal $U$ of $R$, then $R$ is a commutative ring or $F$ is zero on $R$.

\section{Proof:}

According to our hypothesis, we have:

$f(x) \alpha(x)+\alpha(x) f(x)=0$, for all $x \in U$.

The linearization of (1) with respect $x$, we get:

$f(x) \alpha(\omega)+f(\omega) \alpha(x)+2 F(x, \omega) \alpha(x)+2 F(x, \omega)$ $(\omega)+\alpha(x) f(\omega)+2 \alpha(x) F(x, \omega)+(\omega) f(x)+$ $2(\omega) F(x, \omega)=0$, for all $x, \omega \in U$.

Putting $2 x$ instead of $x$ imply that:

$2 f(x) \alpha(\omega)+4 f(\omega) \alpha(x)+4 F(x, \omega) \alpha(x)+8 F(x, \omega)$ $\alpha(\omega)+4 \alpha(x) f(\omega)+4 \alpha(x) F(x, \omega)+2 \alpha(\omega) f(x)+$ $8(\omega) F(x, \omega)=0$, for all $x, \omega \in U$.

Comparing (2) with (3), we arrive because of the 2-torsinity free of $R$ at:

$f(x) \alpha(\omega)+\alpha(\omega) f(x)+2 F(x, \omega) \alpha(x)$

$+2 \alpha(x) F(x, \omega)=0$, for all $x, \omega \in U$.

Replacing $\omega$ by $x \omega$ in (4) leads to:

$f(x) \alpha(x) \alpha(\omega)+\alpha(x) \alpha(\omega) f(x)+2 f(x) \alpha(\omega) \alpha(x)$ $+2 \alpha(x) F(x, \omega) \alpha(x)+2 \alpha(x) f(x) \alpha(\omega)+2 \alpha\left(x^{2}\right)$ $F(x, \omega)=0$, for all $x, \omega \in U$.

Equivalently 
$\alpha(x)(f(x) \alpha(\omega)+\alpha(\omega) f(x)+2 F(x, \omega) \alpha(x)+2 \alpha(x)$ $F(x, \omega))+(f(x) \alpha(x)+\alpha(x) f(x)) \alpha(\omega)$

$+2 f(x)(\omega)(x)=0$, for all $x, \omega \in U$.

In view of (1) and (4), the relation (5) reduces because of the 2-torsinity free of $R$ to:

$f(x) \alpha(\omega) \alpha(x)=0$, for all $x, \omega \in U$.

The substitution $r \omega$ for $\omega$ in (4), we see: $f(x) \alpha(r) \alpha(\omega) \alpha(x)=0$, for all $x, \omega \in U, r \in R$.

Recall that $(U)$ is a nonzero ideal of $R$, also by the primeness of $R$ we can get some $\omega_{0} \in U$ such that $\left(\omega_{0} I\right) \neq\{0\}$, moreover, the automorphisms. It y of $\alpha$ leads to $\omega_{0} I \neq\{0\}$. So there exist $x_{0} \in U$ satisfies that $\omega_{0} x_{0} \neq 0$.

Now, putting $\omega_{0}$ for $\omega$ and $x_{0}$ for $x$ in (6) gives:

$f\left(x_{0}\right) \alpha(r) \alpha\left(\omega_{0} x_{0}\right)=0$, for some $x_{0}, \omega_{0} \in U$ and all $r \in R$.

Using the primeness of $R$, since $\left(\omega_{0} x_{0}\right) \neq 0$, we conclude that $f\left(x_{0}\right)=0$.

Therefore

$f(x)=0$, for all $x$ satisfies that $\omega_{0} x \neq 0$.

Our next task is to prove that $f(x)=0$, for all $x \in U$.

Choose $x \in U$ such that $\omega_{0} x=0$, then

$\omega_{0}\left(x+x_{0}\right) \neq 0$ and $\omega_{0}\left(x-x_{0}\right) \neq 0$, then an application of (7), we have:

$$
\begin{aligned}
& 0=f\left(x+x_{0}\right)=f(x)+f\left(x_{0}\right)+2 F\left(x, x_{0}\right) \\
& =f(x)+2 F\left(x, x_{0}\right) \\
& 0=f\left(x-x_{0}\right)=f(x)-f\left(x_{0}\right)-2 F\left(x, x_{0}\right) \\
& =f(x)-2 F\left(x, x_{0}\right)
\end{aligned}
$$

Combining (8) with (9), we conclude because of the 2-torsinity free of $R$ that $f(x)=0$.

Hence $f(x)=0$, for all $x \in U$. So an application of Lemma (2.4) we get the assertion of the theorem.

\section{Theorem (3.5):}

Let $R$ be a prime ring of characteristic different from 2 and 3 and $U \neq\{0\}$ be an ideal of $R$. if $D_{1}, \quad D_{2}: R \times R \rightarrow R$ are nonzero symmetric $(\alpha, \alpha)$-Biderivations with trace $f_{1}, f_{2}$ respectively satisfies that $f_{1}(u) f_{2}(u)=0$ for all $u$ $\in U$, then either $f_{2}$ is $\alpha$-commuting on $U$ or $R$ is a commutative ring.

\section{Proof:}

By hypothesis, we have:

$f_{1}(u) f_{2}(u)=0$, for all $u, \omega \in U$.

The linearization of (1) leads to:

$f_{1}(u) f_{2}(\omega)+f_{1}(\omega) f_{2}(u)+2 f_{1}(u) D_{2}(u, \quad \omega)+$ $2 f_{1}(\omega) D_{2}(u, \omega)+2 D_{1}(u, \omega) f_{2}(\omega)+2 D_{1}(u, \omega)$ $f_{2}(u)+4 D_{1}(u, \omega) D_{2}(u, \omega)=0$, for all $u, \omega \in U$.

Putting $-u$ instead of $u$ in above relation gives:

$f_{1}(u) f_{2}(\omega)+f_{1}(\omega) f_{2}(u)+4 D_{1}(u, \omega) D_{2}(u, \omega)$

$=0$, for all $u, \omega \in U$.

The linearization of (1) with respect to $\omega$, we find:

$f_{1}(u) f_{2}(\omega)+f_{1}(u) f_{2}(z)+2 f_{1}(u) D_{2}(\omega, z)+f_{1}(\omega)$ $f_{2}(u)+f_{1}(z) f_{2}(u)+2 D_{1}(\omega, z) f_{2}(u)+4 D_{1}(u, \omega)$

$D_{2}(u, \omega)+4 D_{1}(u, z) D_{2}(u, \omega)+4 D_{1}(u, \omega) D_{2}(u$, z) $+4 D_{1}(u, z) D_{2}(u, z)=0$, for all $u, \omega \in U$.

According to (2), the last relation reduces to:

$2 f_{1}(u) D_{2}(\omega, z)+2 D_{1}(\omega, z) f_{2}(u)+4 D_{l}(u, z)$

$D_{2}(u, \omega)+4 D_{1}(u, \omega) D_{2}(u, z)=0$, for $u, \omega \in U$. find:

Replacing $u$ by $\omega$ in above relation, we

$6 f_{1}(\omega) D_{2}(\omega, z)+6 D_{1}(\omega, z) f_{2}(\omega)=0$.

The substitution $z v$ for $z$ in (3) gives:

$f_{1}(\omega) D_{2}(\omega, z) \alpha(v)+f_{1}(\omega) \alpha(z) D_{2}(\omega, v)$

$+D_{1}(\omega, z) \alpha(v) f_{2}(\omega)+\alpha(z) D_{1}(\omega, v) f_{2}(\omega)=0$.

In view of (3), the above relation can be written as:

$D_{1}(\omega, z)\left[\alpha(v), f_{2}(\omega)\right]+\left[f_{1}(\omega), \alpha(z)\right] D_{2}(\omega, v)$ $=0$, for all $v, z, \omega \in U$.

Putting $(z) f_{1}(\omega)$ instead of $\alpha(z)$ yields that:

$D_{1}(\omega, z)\left[\alpha(v), f_{2}(\omega)\right]+\left[f_{1}(\omega), \alpha(z)\right] f_{1}(\omega)$

$D_{2}(\omega, v)=0$, for all $v, z, \omega \in U$.

The substitution $\omega$ for $v$ and using (1) leads to:

$D_{1}(\omega, z)\left[\alpha(\omega), f_{2}(\omega)\right]=0$, for all $z, \omega \in U$. 
Putting $u z$ for $z$ in (4), using (4) implies that:

$D_{1}(\omega, u) \alpha(z)\left[\alpha(\omega), f_{2}(\omega)\right]=0$, for all $z, u, \omega$ $\in U$.

Again, replace $z$ by $z r$ in the last relation leads to:

$D_{1}(\omega, u) \alpha(z) \alpha(r)\left[\alpha(\omega), f_{2}(\omega)\right]=0$, for all $z, u, \omega \in U$ and $r \in R$.

Now, define

$\mathcal{H}=\left\{\omega \in U:\left[\alpha(\omega), f_{2}(\omega)\right]=0\right\}$

$\mathcal{K}=\left\{\omega \in U: D_{l}(\omega, u) \alpha(z)=0\right.$, for all $\left.u, z \in U\right\}$

Since a group cannot be the set theoretic union of two it's proper subgroups, hence either $U=\mathcal{H}$ or $U=\mathcal{K}$. If $U=\mathcal{H}$, this leads that $f_{2}$ is $\alpha$-commuting on $U$. Otherwise, $U=\mathcal{K}$, that is:

$D_{l}(\omega, u) \alpha(z)=0$, for all $z, u, \omega \in U$.

Putting $s z$ instead of $z$, we find:

$D_{l}(\omega, u) \alpha(s) \alpha(z)=0$, for all $z, u, \omega \in U$ and $s \in R$.

By the primeness of $R$, we have either $f_{2}$ is $\alpha$-commuting on $U$ or:

$D_{1}(\omega, u) \alpha(s) \alpha(z)=0$, for all $z, u, \omega \in U$ and $s \in R$.

Since $\alpha$ is an automorphisms, then by the primeness of $R$ (Recall that $(U)$ is a nonzero ideal of $R$ ), we find that $D_{1}(\omega, u)=0$, for all $u, \omega \in U$. Consequently by Lemma (2.3) we conclude that $R$ is commutative.

In similar manner we can prove:

\section{Theorem (3.6):}

Let $R$ be a non-commutative prime ring of characteristic different from 2 and 3 and $U \neq\{0\}$ be an ideal of $R$. if $D_{1}, D_{2}: R \times R \rightarrow R$ are nonzero symmetric $(\alpha, \alpha)$-Biderivations with trace $f_{1}, f_{2}$ respectively satisfies that $f_{1}(u) f_{2}(u)=0$ for all $u \in U$, then either $f_{1}$ is $\alpha$ commuting or $D_{2}$ is a zero mapping on $R$.

\section{Theorem (3.7):}

Let $R$ be a semiprime ring of characteristic different from 2, 3 and $\alpha$ is an automorphism on $R$. if a symmetric left $\alpha$-Bimultiplier $F$ : $R \times R \rightarrow R$ satisfies that $[[f(x), \alpha(x)], \alpha(x)]$ is a central, where $f$ is the Trace of $F$, then $f$ is $\alpha$ commuting on $R$.

\section{Proof:}

For any $x \in R$, we have:

$[[f(x), \alpha(x)], \alpha(x)] \in Z(R)$.

The linearization of (1) leads to:

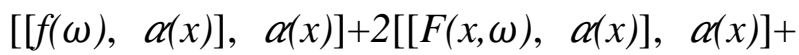
$[[f(\omega), \alpha(\omega)], \alpha(x)]+2[[F(x, \omega), \alpha(\omega)], \alpha(x)]+$ $[[f(x), \quad \alpha(x)], \quad \alpha(\omega)]+[[f(\omega), \quad \alpha(x)], \quad \alpha(\omega)]+$ $2[[F(x, \omega), \alpha(x)], \alpha(\omega)]+[[f(x), \alpha(\omega)], \alpha(\omega)]+$ $[[f(x),(\omega)], \alpha(x)]+2[[F(x, \omega), \alpha(\omega)]$,

$(\omega)] \in Z(R)$, for all $x, \omega \in R$.

The substitution $-x$ for $x$ in (2), then combining the relation so obtained with (2), we arrive because of the 2-torsionity free of $R$ at:

$2[[F(x, \omega), \alpha(x)], \alpha(x)]+[[f(x), \alpha(\omega)], \alpha(x)]+$ $[[f(\omega), \alpha(\omega)], \alpha(x)]+[[f(x), \alpha(x)], \alpha(\omega)]+$ $[[f(\omega), \alpha(x)], \alpha(\omega)]+2[[F(x, \omega), \alpha(\omega)]$,

$(\omega)] \in Z(R)$, for all $x, \omega \in R$.

Also, putting $2 x$ instead of $x$ in (3), we get:

$16[[F(x, \omega), \alpha(x)], \alpha(x)]+8[[f(x), \alpha(\omega)], \alpha(x)]$ $+2[[f(\omega), \alpha(\omega)], \alpha(x)]+8[[f(x), \alpha(x)], \alpha(\omega)]$

$+2[[f(\omega), \alpha(x)], \alpha(\omega)]+4[[F(x, \omega), \alpha(\omega)]$,

$(\omega)] \in Z(R)$, for all $x, \omega \in R$.

Comparing (4) with (3), leads because of the 2-torsinity free of $R$ to:

$2[[F(x, \omega), \alpha(x)], \alpha(x)]+[[f(x), \alpha(\omega)], \alpha(x)]+$ $[[f(x), \alpha(x)], \alpha(\omega)] \in Z(R)$, for all $x, \omega \in R$.

Replacing $\omega$ by $x^{2}$ in (5) and using the commutator identity, we see:

$[[f(x), \alpha(x)], \alpha(x)] \alpha(x)+\alpha(x)[[f(x), \alpha(x)], \alpha(x)]$ $+\alpha(x)[[f(x), \alpha(x)], \alpha(x)]+[[f(x), \alpha(x)], \alpha(x)]$ $a(x)+[[f(x), \alpha(x)], \alpha(x)] \alpha(x)+\alpha(x)[[f(x)$, $\alpha(x)], \alpha(x)] \in Z(R)$, for all $x \in R$.

In view of (1), since $R$ is of characteristic different from 2 and 3 , we can get:

[[f(x), $\alpha(x)], \alpha(x)] \alpha(x) \in Z(R)$, for all $x \in R$.

So for any $u \in R$, we have:

$(u)[[f(x), \alpha(x)], \alpha(x)] \alpha(x)-[[f(x), \alpha(x)], \alpha(x)]$ $\alpha(x)(u)=0$, for all $x \in R$.

According to (1), the above relation can be written as: 
$[[f(x), \alpha(x)],(x)][(u), \alpha(x)]=0$, for $x, u \in R$.

Putting $(u)[f(x), \alpha(x)]$ instead of $\alpha(u)$ in (6), using (6), leads to:

$[[f(x), \alpha(x)], \alpha(x)](u)[[f(x), a(x)], \alpha(x)]=0$, for all $x, u \in R$.

Using the semiprimeness of $R$ and automorphismity of $\alpha$, we conclude that:

$[[f(x), \alpha(x)], \alpha(x)]=0$, for all $x \in R$.

Now, using a same argument on (7) as used to get (5) from (1), we can see:

$[[f(x),(\omega)], \alpha(x)]+[[f(x), \alpha(x)],(\omega)]+$ $2[[F(x, \omega), \alpha(x)], \alpha(x)]=0$, for $x, \omega \in R$.

Replacing $\omega$ by $v \omega$ in (8) gives:

$[(\omega)[f(x), \quad \alpha(v)], \quad \alpha(x)]+[[f(x), \quad \alpha(\omega)] \quad \alpha(v)$, $\alpha(x)]+[[f(x), a(x)], \alpha(\omega)] \alpha(v)+\alpha(\omega)[[f(x)$, $\alpha(x)], \alpha(v)]+2[[F(x, \omega) \alpha(v), \alpha(x)], \alpha(x)]=0$.

That is for all $x, v, \omega \in R$, we have:

$[(\omega), \quad \alpha(x)] \quad[f(x), \quad \alpha(v)]+(\omega)[[f(x), \quad \alpha(v)]$, $\alpha(x)]+[f(x), \alpha(\omega)][\alpha(v), \alpha(x)]+[[f(x), \alpha(\omega)]$, $\alpha(x)] \alpha(v)+[[f(x), \alpha(x)], \alpha(\omega)] \alpha(v)+\alpha(\omega)[f(x)$, $\alpha(x)], \alpha(v)]+2[F(x, \omega), \alpha(x)][\alpha(v), \alpha(x)]+$ $2 F(x, \omega)[[\alpha(v), \alpha(x)], \alpha(x)]+2[[F(x, \omega), \alpha(x)]$, $\alpha(x)] \alpha(v)+2[F(x, \omega), \alpha(x)][\alpha(v), \alpha(x)]=0$,

An application of (8), the above relation reduces to:

$[f(x), \alpha(\omega)][(v), \alpha(x)]+[(\omega), \alpha(x)][f(x),(v)]$ $+4[F(x, \omega), \quad \alpha(x)][(v), \quad \alpha(x)]+2 F(x, \omega)$ $[[\alpha(v), \alpha(x)], \alpha(x)]=0$, for all $x, v, \omega \in R$.

The substitution $x$ for $v$ in (9) imply that: $[(\omega), \alpha(x)][f(x), \alpha(x)]=0$, for all $x, \omega \in R$.

Putting $f(x)(\omega)$ instead of $\alpha(\omega)$ in (10), then using (10) gives:

$[f(x), \alpha(x)](\omega)[f(x), \alpha(x)]=0$, for all $x, \omega \in R$.

The semiprimeness of $R$ leads to:

$[f(x), \alpha(x)]=0$, for all $x, \omega \in R$.

Hence $f$ is an $\alpha$-commuting mapping on $R$.

We end this paper with the following result which gives a suitable condition on asymmetric generalized $(\alpha, \alpha)$-Biderivation $\mathrm{G}$ : $R \times R \rightarrow R$ that makes the ring $R$ is a commutative.

\section{Theorem (3.8):}

Let $R$ be a 2 -torision free prime ring and $U$ be a nonzero ideal of $R$. if a symmetric generalized $(\alpha, \alpha)$-Biderivation $G: R \times R \rightarrow R$ with associated $(\alpha, \alpha)$-Biderivation $D$ satisfies that $G(d(u), v)=0$ for all $u, v \in U$ where $d$ is the Trace of $D$, then $D$ is a zero mapping on $R$.

\section{Proof:}

By hypothesis, we have:

$G(d(u), v)=0$, for all $u, v \in U$. that:

Replacing $v$ by $v z$ in above relation implies $G(d(u), v) \alpha(z)+\alpha(v) D(d(u), z)=0$, for $u, v, z$ $\in U$.

According to (1), the above relation reduces to:

$D(d(u), z)=0$, for all $u, z \in U$.

The substitution $u+v$ for $u$ in (2) give:

$D(d(u), z)+D(d(v), z)+2 D(D(u, v), z)=0$, for all $u, v \in U$.

According to (2), the last relation becomes: $2 D(D(u, v), z)=0$, for all $u, v, z \in U$.

Putting $v \omega$ instead of $v$ in (3), we get:

$2 D(D(u, v) \alpha(\omega)+\alpha(v) D(u, \omega), z)=0$, for all $u, v, z, \omega \in U$.

Equivalently

$2 D(D(u, v), z) \alpha^{2}(\omega)+2 \alpha(D(u, v) D(\alpha(\omega), z)$ $+2 D(\alpha(v), z) \alpha\left(D(u, \omega)+2 \alpha^{2}(v) D(D(u, \omega), z)\right.$ $=0$, for all $u, v, z, \omega \in U$.

An application of (3) on above relation leads to:

$D(\alpha(v), z) \alpha(D(u, \omega)+\alpha(D(u, v)) D(\alpha(\omega), z)$

$=0$, for all $u, v, z, \omega \in U$.

Replacing $v$ by $v k$ in (4) implies that: 
$\alpha\left(D(u, v) \alpha^{2}(k) D(\alpha(\omega), z)+\alpha^{2}(v) \alpha(D(u\right.$, $k) D(\alpha(\omega), z)+\alpha^{2}(v) D(\alpha(k), z) \alpha(D(u, \omega))+$

$D(\alpha(v), z) \alpha^{2}(k) \alpha(D(u, \omega)=0$, for $u, v, z, k, \omega$ $\in U$.

In view of (4), the above relation becomes:

$\alpha(D(u, v)) \alpha^{2}(k) D(\alpha(\omega), z)+D(\alpha(v), z) \alpha^{2}(k)$ $\alpha(D(u, \omega))=0$, for all $u, v, z, k, \omega \in U$.

Putting $u$ for $z$ and $\omega$ for $v$ in above relation, we find:

$\alpha(D(u, \omega)) \alpha^{2}(k) D(\alpha(\omega), u)+D(\alpha(\omega), u) \alpha^{2}(k)$ $\alpha(D(u, \omega))=0$, for all $u, k, \omega \in U$.

Recall that $(U)$ is an ideal of $R$, replace $(\omega)$ by $\omega$, then an application of Lemma (2.1) on above relation yields because of automorphismity of $\alpha$ and symmetry of $D$ that:

$D(u, \omega)=0$, for all $u, \omega \in U$.

Using Lemma (3.2), we get the requirements of the theorem.

\section{References}

[1] Lee T. K., " $\alpha$-commuting mappings in semiprime rings", Communications in Algebra (29), 2945-2951, (2001).

[2] Maksa G., "Remark on symmetric biadditive functions having non-negative diagonalization", Glasnik Math. 15, 279280, (1980).

[3] Ceven Y., and Öztürk M. A., "Some properties of symmetric $\operatorname{Bi}-(\sigma, \tau)$ Derivations in Near-Rings", Commun. Korean Math. Soc. 22, No. 4, 487-491, (2007).

[4] Ashraf M., "On Symmetric Generalized $(\alpha, \beta)$-Deriavations in Rings", International Congress of mathematicians, Hyderabad, India, 19-27, August (2010).

[5] Bell H. E. and Martindale W. S., "Centralizing mappings of Semiprime Rings", Cand. Math. Bull. 30, 92-101, (1987).

[6] Brešar M. and Vukman J., "On the left derivations and related mappings", Proc. Amer. Math. Soc., 110, 19-27, (1990).

[7] Brešar M., Martindale W. S. III and Miers C. R., "Centralizing maps in prime rings with involution", J. Algebra, 161, 332-357, (1993).

[8] Serif Y. M. and Argac N., "Ideals and Symmetric Bi-derivation of prime and Semi-prime Rings", Math J. Okayama Univ. Vol. 35, (1), 189-192, (1993).

[9] Herestein I. N., "Rings with involution", The University of Chicago Press, Chicago, (1976).

[10] Auday H. M., “On Generalized Bi derivations and related additive mappings", Ph.D. Thesis, University of Almustansiriya, Iraq, (2015).

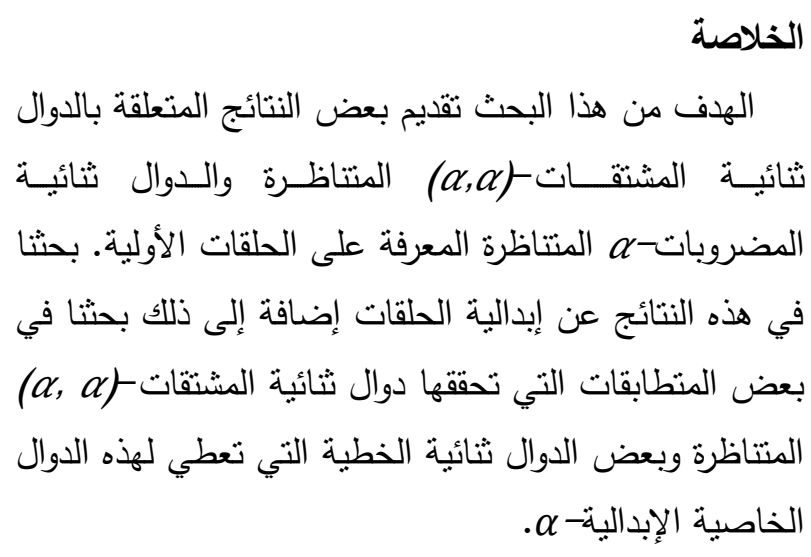

